\title{
COMPUTATIONAL METHODOLOGY TO ANALYZE THE EFFECT OF MASS TRANSFER RATE ON ATTENUATION OF LEAKED CARBON DIOXIDE IN SHALLOW AQUIFERS
}

\author{
Radek Fučík ${ }^{a, *}$, Jakub SolovskÝ ${ }^{a}$, Michelle R. Plampin ${ }^{b}, \mathrm{HaO} \mathrm{Wu}^{c}$, \\ JiŘÍ MIKYŠKA $^{a}$, TISSA H. ILlANGASEKARE ${ }^{d}$ \\ ${ }^{a}$ Czech Technical University in Prague, Faculty of Nuclear Sciences and Physical Engineering, Department of \\ Mathematics, Trojanova 13, 12000, Praha, Czech Republic \\ ${ }^{b}$ U.S. Geological Survey, Eastern Energy Resources Science Center, 12201 Sunrise Valley Drive, Reston, VA \\ 20192, USA \\ ${ }^{c}$ Virginia Polytechnic Institute and State University, Department of Geosciences, 926 West Campus Drive, \\ Blacksburg, VA 24061, USA \\ ${ }^{d}$ Colorado School of Mines, Center for Experimental Study of Subsurface Environmental Processes, 1500 Illinois \\ St., Golden, CO 80401, USA \\ * corresponding author: fucik@fjfi.cvut.cz
}

\begin{abstract}
Exsolution and re-dissolution of $\mathrm{CO}_{2}$ gas within heterogeneous porous media are investigated using experimental data and mathematical modeling. In a set of bench-scale experiments, water saturated with $\mathrm{CO}_{2}$ under a given pressure is injected into a 2-D water-saturated porous media system, causing $\mathrm{CO}_{2}$ gas to exsolve and migrate upwards. A layer of fine sand mimicking a heterogeneity within a shallow aquifer is present in the tank to study accumulation and trapping of exsolved $\mathrm{CO}_{2}$. Then, clean water is injected into the system and the accumulated $\mathrm{CO}_{2}$ dissolves back into the flowing water. Simulated exsolution and dissolution mass transfer processes are studied using both nearequilibrium and kinetic approaches and compared to experimental data under conditions that do and do not include lateral background water flow. The mathematical model is based on the mixed hybrid finite element method that allows for accurate simulation of both advection- and diffusion-dominated processes.
\end{abstract}

Keywords: Compositional flow, two-phase flow, kinetic mass transfer, gas exsolution, gas dissolution.

\section{INTRODUCTION}

Geologic carbon sequestration has the potential to significantly reduce greenhouse gas emissions [1], but also poses risks to groundwater resources including mobilization of contaminants in shallow aquifers due to leakage of $\mathrm{CO}_{2}$ from deep storage formations [2]. The extent and severity of the risks depend on complex multiphase flow and transport phenomena that govern the migration of $\mathrm{CO}_{2}$ through the shallow subsurface. A persistent issue with predicting these processes is the general difficulty of understanding $\mathrm{CO}_{2}$ phase change (a.k.a. inter-phase mass transfer) within macroscopic porous media systems, which is important in the case of $\mathrm{CO}_{2}$ due to its high solubility and potential mobility in the gas phase.

If a leakage pathway is encountered, stored $\mathrm{CO}_{2}$ that is originally supercritical in a deep geologic storage formation may migrate upward due to buoyancy, dissolve into water, exsolve to form a separate gas phase, and eventually re-dissolve into clean water. These interrelated processes are collectively referred to as multiphase evolution, and have recently been studied in various continuum-scale systems. Experimental investigations have identified the factors that control multiphase evolution during one-dimensional (1D) ver- tical flow 3-5] and have qualitatively addressed the various transport phenomena during two-dimensional (2D) flow 6. Numerical modeling has been used to show the effects of $1 \mathrm{D}$ flow rate [7] and quasi-2D flow paths [8] on multiphase $\mathrm{CO}_{2}$ evolution processes. However, numerical models have not yet been able to fully explain all of the observations from the experimental studies, particularly of those that occur during $2 \mathrm{D}$ flow under the influence of background water flow.

The purpose of this present study is to develop a numerical modeling methodology to assess the factors that control inter-phase $\mathrm{CO}_{2}$ mass transfer during migration through heterogeneous $2 \mathrm{D}$ porous media systems in the shallow subsurface where $\mathrm{CO}_{2}$ can only exist in the gaseous and dissolved phases. Specifically, we seek to test whether the local equilibrium assumption for mass transfer adequately explains multiphase evolution, and to investigate the effect of lateral water flow on heterogeneity-enhanced gas phase accumulation.

Our approach is to expand the numerical model developed in [9] to include kinetic mass transfer, and then to compare the results of the simulations performed by the model to the laboratory data from experiments that build upon methods developed in 
[10. This methodology forms the basis for another article [1] that investigates multiphase $\mathrm{CO}_{2}$ plume dynamics in relatively large-scale synthetic aquifer systems. These investigations improve our understanding of the underlying processes that together lead to attenuation of $\mathrm{CO}_{2}$ within groundwater systems [6].

The main objective of this work is to study the exsolution and dissolution of $\mathrm{CO}_{2}$ on a small scale using both experiments and numerical modeling. Motivated by fundamental differences in mass transfer in various scenarios studied in [11, our aim is to determine whether a kinetic or a simplified equilibrium mass transfer model is needed to reproduce the results observed in the experiments and how the results are affected by changes in the flow field enforced in the experiments.

\section{EXPERIMENT}

The experimental methodology is detailed by [10, so it will be only briefly described here. To assess the effects of background (lateral) water flow on multiphase $\mathrm{CO}_{2}$ migration, two different bench-scale experiments were performed. In each experiment, $\mathrm{CO}_{2}$-saturated water was injected into the bottom of a tank filled with water-saturated porous media until gas phase formed and accumulated to some steady value. Then, clean water was injected until all of the $\mathrm{CO}_{2}$ dissolved and migrated out of the tank.

The test system was initially reported by [10, but this study expands upon that methodology by incorporating different experimental conditions. Most importantly, one experiment was performed with water flowing from right to left across the tank. As is shown in Figure 1, a block of low-permeability sand was incorporated into the middle of the tank above the injection ports, and saturation sensors were installed below this block of fine sand where gas phase $\mathrm{CO}_{2}$ was expected to accumulate after exsolving from the injected $\mathrm{CO}_{2}$-saturated water. Material properties of the three porous media used are given in Table 3 The saturation pressure, as defined by [3], was $10 \mathrm{kPa}$ for the first experiment and $15 \mathrm{kPa}$ for the second, which led to dissolved $\mathrm{CO}_{2}$ concentrations that were sufficiently high to cause exsolution in the porous media.

In the first experiment, the constant head devices connected to the regions of Granusil \#8 (which were installed to distribute the head evenly across the vertical boundaries of the main sand pack) were positioned at equal elevations. This led to negligible background water flow across the tank, and this case is therefore referred to as the static case. In the second experiment, the left hand constant head device was positioned at an elevation below that of the right-hand one, thus establishing background flow. In the second case, water inflow was supplied to the right-hand constant head device via a peristaltic pump, while the outflow from the left-hand constant head device was routed into a container placed on a computer-interfaced electronic scale. The scale and the saturation sensors were configured to automatically take measurements at 1-minute intervals.

The resulting data from the experiments were compared against a numerical model of two-phase flow in porous media, which extends upon that of 9 . A major addition to the model is its capability to account for non-equilibrium $\mathrm{CO}_{2}$ mass transfer between aqueous and gas phases.

\section{Mathematical MOdel}

In this section, the mathematical model that describes the two-phase compositional flow in porous media and incorporates the phenomena studied in this work is summarized.

\subsection{TWO-PHASE FLOW IN POROUS MEDIA}

The governing equations for the two-phase flow in porous media are based on [12 14. The quantities corresponding to the liquid (wetting) and gas (nonwetting) phases are denoted by indices $\ell$ and $g$, respectively.

The mass balance equations for the incompressible liquid and gas phases are given by

$$
\rho_{\ell} \phi \frac{\partial S_{\ell}}{\partial t}+\rho_{\ell} \nabla \cdot \vec{v}_{\ell}=f_{\ell},
$$

and

$$
\rho_{g} \phi \frac{\partial S_{g}}{\partial t}+\rho_{g} \nabla \cdot \vec{v}_{g}=f_{g},
$$

respectively, where $\phi[-]$ is the material porosity and $S_{\alpha}[-], \rho_{\alpha}\left[\mathrm{kg} \mathrm{m}^{-3}\right], \vec{v}_{\alpha}\left[\mathrm{m} \mathrm{s}^{-1}\right], f_{\alpha}\left[\mathrm{kg} \mathrm{m}^{-3} \mathrm{~s}^{-1}\right]$ are the $\alpha$-phase saturation, density, velocity, and the sink or source term. The velocity $\vec{v}_{\alpha}$ is given by Darcy's law

$$
\vec{v}_{\alpha}=-\lambda_{\alpha} K\left(\nabla p_{\alpha}-\rho_{\alpha} \vec{g}\right),
$$

where $\vec{g}\left[\mathrm{~m} \mathrm{~s}^{-2}\right]$ is the gravitational acceleration vector, $K\left[\mathrm{~m}^{2}\right]$ is the intrinsic permeability, $p_{\alpha}[\mathrm{Pa}]$ is the $\alpha$-phase pressure, $\lambda_{\alpha}=k_{r_{\alpha}} / \mu_{\alpha}\left[\mathrm{Pa}^{-1} \mathrm{~s}^{-1}\right]$ denotes the mobility of phase $\alpha$, where $\mu_{\alpha}[\mathrm{Pa} \mathrm{s}]$ is the dynamic viscosity, and $k_{r \alpha}\left(S_{\alpha}\right)[-]$ denotes the relative permeability.

The difference between the wetting and non-wetting phase pressures is defined as the capillary pressure $p_{c}=p_{g}-p_{\ell}$ and the Brooks and Corey model [15] is used in the form

$$
p_{c}\left(S_{\ell}\right)=p_{d}\left(S_{\ell}^{e}\right)^{-\frac{1}{\lambda}}
$$

where $p_{d}[\mathrm{~Pa}]$ is the entry pressure, $\lambda[-]$ is the pore size distribution index, and $S_{\alpha}^{e}[-]$ denotes the effective saturation defined by

$$
S_{\alpha}^{e}=\frac{S_{\alpha}-S_{r, \alpha}}{1-S_{r, g}-S_{r, \ell}},
$$

where $S_{r, \alpha}[-]$ is the residual saturation of phase $\alpha$, $\alpha \in\{\ell, g\}$. 


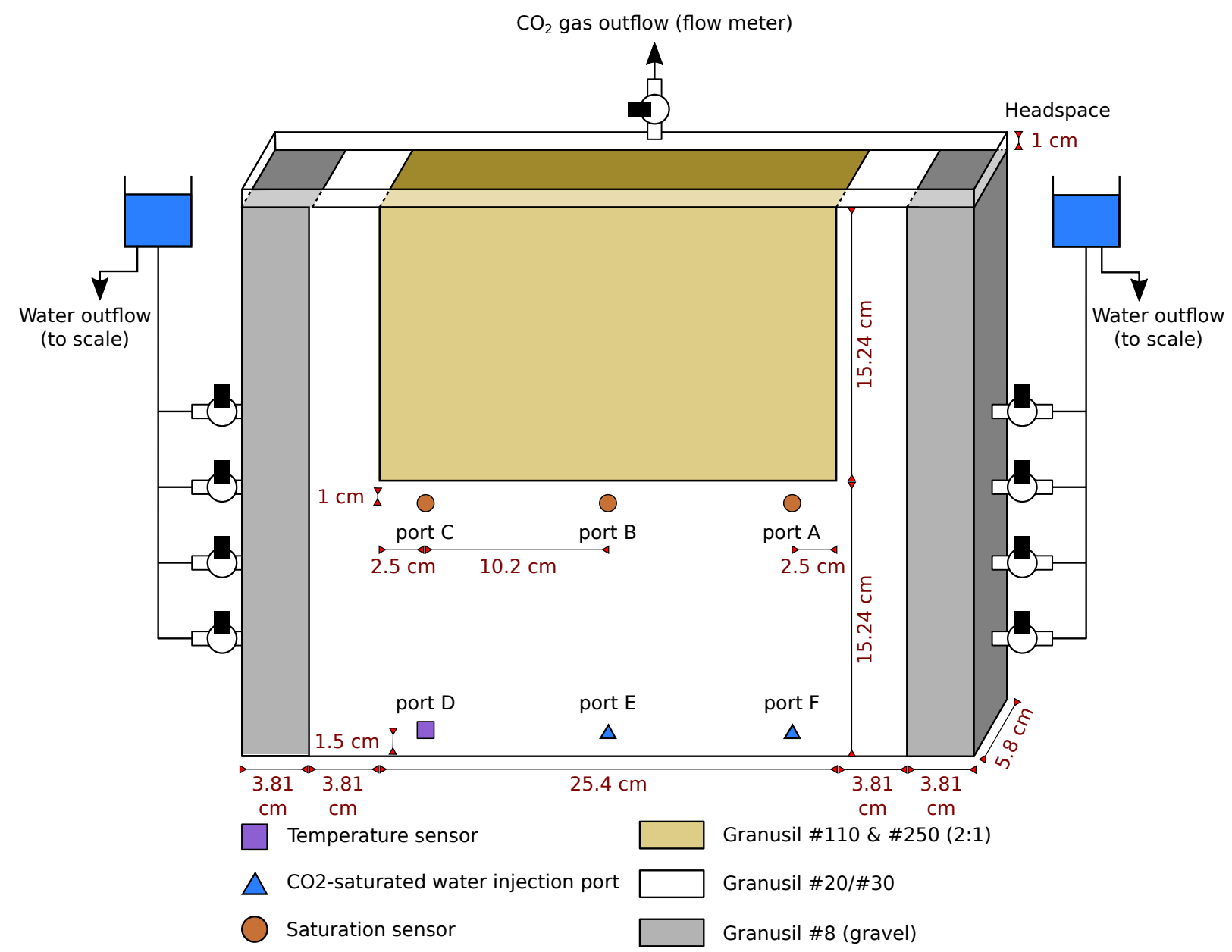

Figure 1. Setup for the small tank experiments, adapted from [10].

The Brooks and Corey model parameters are also used in the Burdine's model for the relative permeability functions $k_{r, \ell}$ and $k_{r, g},[16$, in the form

$$
\begin{aligned}
& k_{r, \ell}\left(S_{\ell}\right)=\left(S_{\ell}^{e}\right)^{\frac{2+3 \lambda}{\lambda}} \\
& k_{r, g}\left(S_{g}\right)=\left(S_{g}^{e}\right)^{2}\left(1-\left(1-S_{g}^{e}\right)^{\frac{2+\lambda}{\lambda}}\right) .
\end{aligned}
$$

Based on [7, 11, 17, however, instead of $k_{r, g}$, a modified formula for the gas phase relative permeability function is used in Eq. (2) in the form

$$
\tilde{k}_{r, g}\left(S_{g}\right)=\left\{\begin{aligned}
0, & \text { if } S_{g}<S_{c}, \\
k_{r, g}\left(\frac{S_{g}-S_{c}}{1-S_{c}}\right), & \text { otherwise }
\end{aligned}\right.
$$

where $S_{c}[-]$ denotes the critical gas saturation.

\subsection{Component transport}

The gas phase is considered as a single component (pure $\mathrm{CO}_{2}$ ), whereas the liquid phase is assumed to be a two component mixture (water and $\mathrm{CO}_{2}$ ). The compositional balance equation for $\mathrm{CO}_{2}$ dissolved in the liquid phase is included into (1) and (2) as

$$
\phi \rho_{\ell} \frac{\partial\left(S_{\ell} X\right)}{\partial t}+\rho_{\ell} \nabla \cdot\left(X \vec{v}_{\ell}-\tau \phi S_{\ell} D_{\ell} \nabla X\right)=f_{X},
$$

[14], where $X[-]$ is the mass fraction of $\mathrm{CO}_{2}$, $f_{X} \quad\left[\begin{array}{lll}\mathrm{kg} & \mathrm{m}^{-3} & \mathrm{~s}^{-1}\end{array}\right]$ is the sink or source term, $D_{\ell}\left[\mathrm{m}^{2} \mathrm{~s}^{-1}\right]$ is the free molecular diffusion of $\mathrm{CO}_{2}$ in water, $D_{\ell}=1.92 \cdot 10^{-9} \mathrm{~m}^{2} \mathrm{~s}^{-1}$, and $\tau_{\ell}[-]$ is the tortuosity given by $\tau_{\ell}=\phi^{1 / 3} S_{\ell}^{7 / 3}$ based on [18].

\subsection{KinetiC MASS TRANSFER}

Based on [19], the kinetic mass transfer of $\mathrm{CO}_{2}$ between both phases (i.e., the dissolution and exsolution processes) is represented by

$$
-f_{g}=f_{\ell}=f_{X}=k\left(C_{s}-X \rho_{\ell}\right),
$$

where $k\left[\mathrm{~s}^{-1}\right]$ is the lumped mass transfer rate coefficient and $C_{s}\left[\mathrm{~kg} \mathrm{~m}^{-3}\right]$ is the saturated $\mathrm{CO}_{2}$ concentration in water at the relevant pressure and temperature given by Henry's law in the form

$$
C_{s}=\frac{p_{g}}{K_{H}} M_{g},
$$


where $M_{g}\left[\mathrm{~kg} \mathrm{~mol}^{-1}\right]$ is the molar mass of $\mathrm{CO}_{2}, M_{g}=$ $44.01 \mathrm{~g} \mathrm{~mol}^{-1}$, and $K_{H}\left[\mathrm{~Pa} \mathrm{~mol}^{-1} \mathrm{~m}^{3}\right]$ is Henry's constant for which the Van't Hoff equation is employed in the form

$$
K_{H}=K_{H, r e f} e^{-C\left(\frac{1}{T}-\frac{1}{T_{r e f}}\right)}
$$

where $T[K]$ is the temperature, $K_{H, r e f}$ is the value of Henry's constant at a reference temperature $T_{\text {ref }}[K]$, and $C[K]$ is the gas-specific constant, i.e., $K_{H, r e f}=$ $2979.97\left[\mathrm{~Pa} \mathrm{~mol}^{-1} \mathrm{~m}^{3}\right], T_{\text {ref }}=298.15 \mathrm{~K}$, and $C=$ $2400 \mathrm{~K}$ [20]. The average temperatures in the static case and background flow experiments were $37^{\circ} \mathrm{C}$ and $26^{\circ} \mathrm{C}$, respectively.

\section{Numerical METHOD AND IMPLEMENTATION}

The governing equations are solved using a general numerical solver based on the mixed-hybrid finite element method described in [9]. The mixed-hybrid finite element method combines velocity discretizations in the lowest order Raviart-Thomas-Nédélec space with piecewise constant approximations for the scalar variables. The numerical method can be used for accurate simulation of degenerate diffusion or advectiondominated problems like the one discussed here.

\subsection{NumericAL METHOD}

The numerical method solves a system of $n$ partial differential equations in the coefficient form in a $d$ dimensional polygonal domain $\Omega \subset \mathbb{R}^{d}$ and a time interval $\left[0, t_{\text {fin }}\right]$ :

$$
\begin{aligned}
\sum_{j=1}^{n} N_{i, j} \frac{\partial Z_{j}}{\partial t}+ & \sum_{j=1}^{n} \vec{u}_{i, j} \cdot \nabla Z_{j}+ \\
& \nabla \cdot\left(m_{i}\left(\vec{q}_{i}+\vec{w}_{i}\right)\right)=f_{i},
\end{aligned}
$$

with

$$
\vec{q}_{i}=-\sum_{j=1}^{n} D_{i, j} \nabla Z_{j}
$$

where $Z_{j}=Z_{j}(t, \vec{x}), j=1,2, \ldots, n$, represent the unknown variables, $\vec{x} \in \Omega, t \in\left[0, t_{\text {fin }}\right]$. Eq. [12 is further supplemented with Dirichlet or Neumann boundary conditions, or its combination on different parts of the boundary [9].

Here we restrict ourselves only the case $d=2$ which is relevant for the problems presented in the paper. In brevity, the mixed hybrid finite element discretization approximates the unknown scalar functions $Z_{j}$ in the space of piecewise constant functions, i.e.,

$$
Z_{j} \approx \sum_{A \in \mathcal{A}_{h}} Z_{j, A} \varphi_{A}
$$

and the vector function $\vec{q}_{i}$ from Eq. $12 \mathrm{~b}$ in the lowest order Raviart-Thomas-Nédélec space $\mathrm{RTN}_{0}$ as

$$
\vec{q}_{i} \approx \sum_{A \in \mathcal{A}_{h}} \chi_{A} \sum_{E \in \mathcal{E}_{A}} q_{i, A, E} \vec{\omega}_{A, E}
$$

where $\mathcal{A}_{h}$ denotes the set of triangles discretizing the computational domain $\Omega, h$ is the mesh size defined as the largest ball diameter circumscribed around elements in $\mathcal{A}_{h}, \mathcal{E}_{A}$ is the set of all sides of an element $A \in \mathcal{A}_{h}, \varphi_{A}$ are the piecewise constant basis functions defined by $\varphi_{A}=\chi_{A}$, where $\chi_{A}$ is the characteristic function of $A$ defined by

$$
\chi_{A}(\vec{x})= \begin{cases}1 & \vec{x} \in A, \\ 0 & \vec{x} \notin A,\end{cases}
$$

and $\vec{\omega}_{A, E}$ are the vector basis functions of $R T N_{0}(A)$, [9].

Eq. $12 \mathrm{~b}$ and the discretization given by Eq. 14 allow to express the coefficients $q_{i, A, E}$ as

$$
q_{i, A, E}=\sum_{j \in \sigma_{i, A}}\left(b_{i, j, A, E} Z_{j, A}-\sum_{F \in \mathcal{E}_{A}} b_{i, A, E, F} Z_{j, F}\right),
$$

where $Z_{j, E}$ are traces of $Z_{j}$ on side $E \in \mathcal{E}_{A}, \sigma_{i, A} \subseteq$ $\{1,2, \ldots, n\}$ denotes the set of all indices $j$ for which $D_{i, j}$ is non-zero on element $A$ and the definition of coefficients $b_{i, j, A, E}$ and $b_{i, j, A, E, F}$ are given in 9 .

The approximation of $\vec{q}_{i}$ in $\mathrm{RTN}_{0}$ given by Eqs. (14) and (16) together with the piecewise constant approximation of Eq. (12a) allow to express the cell-averages $Z_{j, A}$ as a local linear combination of $Z_{j, E}, E \in \mathcal{E}_{A}$, on each element $A \in \mathcal{A}_{h}$, see [9] for details. Hence, $q_{i, A . E}$ can be expressed solely as linear function of traces $Z_{j, E}, \forall E \in \mathcal{E}_{A}$.

Across all interior sides $E$ of the triangulation $\mathcal{A}_{h}$, the balance of conservative fluxes is given by

$$
\sum_{\left\{A: E \in \mathcal{E}_{A}\right\}} m_{i, E}\left(\vec{q}_{i, A, E}+\vec{w}_{i, A, E}\right)=0,
$$

where a single (upwinded) value $m_{i, E}$ is assumed at $E$, see [9], and, thus, it can be eliminated from Eq. 17] if it has a non-zero value to produce

$$
\sum_{\left\{A: E \in \mathcal{E}_{A}\right\}} \vec{q}_{i, A, E}+\vec{w}_{i, A, E}=0
$$

When $m_{i, E}=0$ in Eq. (17) for some side E, Eq. (18) is also considered to assure that the resulting system of linear equations, given in the vector form as

$$
M^{k} \vec{Z}^{\ell+1}=\vec{b}^{\ell},
$$

is non-singular. In Eq. (19), $\boldsymbol{M}$ is a sparse, positive definite matrix [9] and $\vec{b}$ is the right-hand-side, both evaluated on the previous time level $\ell$, and $\vec{Z}$ is the vector containing side-traces $Z_{j, E}$ on the next time level $\ell+1$ for all $j=1,2, \ldots, n$ and all sides $E$ in $\mathcal{A}_{h}$.

\subsection{Problem formulation}

The system of governing equations given by Eqs. (1), (2), (8), and (9) is represented by (12) using $n=3$, 


\begin{tabular}{lcccc}
\hline Parameter & & Units & $\begin{array}{c}\text { liquid } \\
\mathrm{H}_{2} \mathrm{O}\end{array}$ & $\begin{array}{c}\text { gas } \\
\mathrm{CO}_{2}\end{array}$ \\
\hline Density & $\rho$ & {$\left[\mathrm{kg} \mathrm{m}^{-3}\right]$} & 997.78 & 1.98 \\
Dyn. viscosity & $\mu$ & {$\left[10^{-5} \mathrm{~Pa} \mathrm{~s}^{-1}\right]$} & 87.2 & 1.48 \\
\hline
\end{tabular}

TABLE 1. Properties of fluids.

\begin{tabular}{lcccc}
\hline & Units & Mesh & Mesh & Mesh \\
& & $\Delta_{1}$ & $\Delta_{2}$ & $\Delta_{3}$ \\
\hline Elements & & 5494 & 18587 & 72949 \\
Mesh size $h$ & {$[\mathrm{~mm}]$} & 5.55 & 2.78 & 1.43 \\
\hline
\end{tabular}

TABLE 2. Properties of meshes.

$$
\begin{aligned}
& d=2, Z_{1}= p_{c}, Z_{2}=p_{g}, Z_{3}=X, \text { and } \\
&\left(N_{i, j}\right)_{i, j \in \hat{3}}=\left(\begin{array}{ccc}
-\phi \rho_{\ell} \frac{d S_{\ell}}{d p_{c}} & 0 & 0 \\
-\phi \rho_{g} \frac{d S_{\ell}}{d p_{c}} & 0 & 0 \\
0 & 0 & \phi S_{\ell} \rho_{\ell}
\end{array}\right), \\
&\left(\vec{u}_{i, j}\right)_{i, j \in \hat{3}}=\left(\begin{array}{ccc}
\overrightarrow{0} & \overrightarrow{0} & \overrightarrow{0} \\
\overrightarrow{0} & \overrightarrow{0} & \overrightarrow{0} \\
\overrightarrow{0} & \overrightarrow{0} & \rho_{\ell} \vec{\vartheta}_{\ell}
\end{array}\right), \\
&\left(m_{i}\right)_{i \in \hat{3}}=\left(\begin{array}{c}
\rho_{\ell} \frac{\lambda_{\ell}}{\lambda_{\ell}+\lambda_{g}} \\
\rho_{g} \frac{\lambda_{\ell}}{\lambda_{\ell}+\lambda_{g}} \\
\rho_{\ell}
\end{array}\right), \\
&\left(D_{i, j}\right)_{i, j \in \hat{3}}=\left(\begin{array}{cc}
\left(\lambda_{\ell}+\lambda_{g}\right) K & -\lambda_{\ell}+\lambda_{g} K \\
0 & \left(\lambda_{\ell}+\lambda_{g}\right) K \\
0 & 0 \\
\left(\vec{w}_{i}\right)_{i \in \hat{3}} & =\left(\begin{array}{c}
-\left(\lambda_{\ell}+\lambda_{g}\right) \rho_{\ell} K \vec{g} \\
\lambda_{\ell}+\lambda_{g} \rho_{g} K \vec{g} \\
\overrightarrow{0} \\
-f_{\ell} \\
f_{g} \\
f_{\ell}-X f_{\ell}
\end{array}\right),
\end{array}\right.
\end{aligned}
$$

where $\hat{3}=\{1,2,3\}$.

The computational domain $\Omega$ depicted in Figure 2 is discretized using three gradually refined triangular meshes generated by gmsh [21]. The mesh properties are listed in Table 2 and in Figure 2, the coarsest mesh is shown.

The numerical method is implemented in an inhouse computer code using C++. The applicability of the numerical method for heterogeneous porous media is further discussed in 22 together with parallel implementations of the method on GPU 9 or on CPU using MPI [23].

\section{Results AND Discussion}

\subsection{Computational STudy}

After the physical domain and boundaries were set up to mimic the experiments, several simulations were performed for each experimental case (i.e., the static

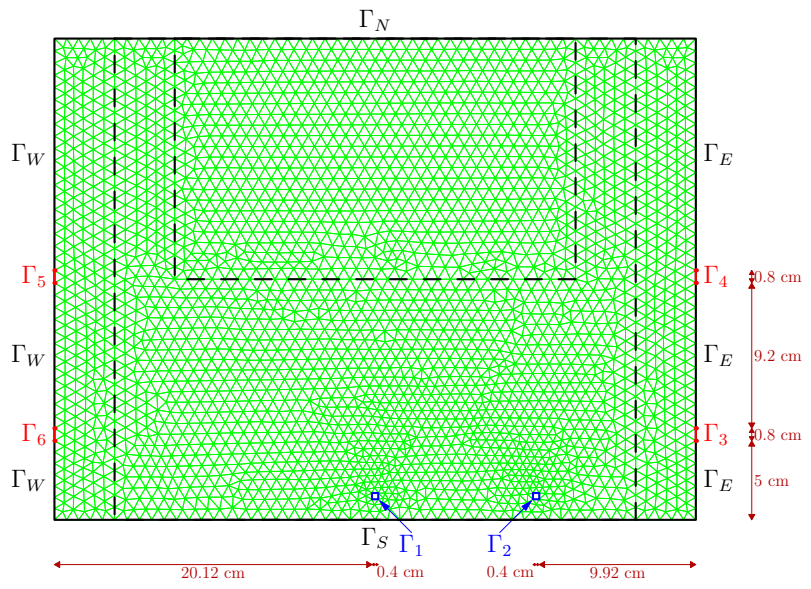

Figure 2. Computational domain with coarse mesh based on Figure 1 .

one and the one with background flow), using several different values for $k$. The boundary conditions on the model included hydrostatic pressure in the inflow/outflow ports on the left and right-hand side of the tank (see Figure 1). For the experiment with background flow, the pressure on the right-hand side was increased to enforce a lateral pressure gradient and thus background flow through the tank. The pressure difference across the tank was fitted to match the measured outflow rates from the experiment. Injection of $\mathrm{CO}_{2}$-saturated and clean water was represented by a Neumann boundary condition at the injection port. No flow boundary conditions were prescribed along the remaining parts of the computational domain boundary.

Several additional simulations were performed to investigate the effect of different background water flow rates on gas accumulation below the heterogeneity. All of these simulations were performed with a high (near-equilibrium) mass transfer rate of $k=1 s^{-1}$. The simulation representing the experiment with background flow was conducted with a pressure difference of $5 \mathrm{~Pa}$, while the other simulations were performed with pressure differences of $2,4,6,7,8$, and $9 \mathrm{~Pa}$.

\subsection{Numerical CONVERGENCE}

The mesh effects on the numerical solution are investigated using three gradually refined triangular meshes described in Table 2. The gas saturation profiles at Ports A, B, and C for $k=0.5 \mathrm{~s}^{-1}$ and $S_{c}=0.2$ for both static and background flow cases are compared in Figure 3. The amount of gas simulated present in ports $\mathrm{A}, \mathrm{B}$, and $\mathrm{C}$ is slightly overestimated for coarser meshes with respect to finer meshes, however, these results are expected due to the first order of convergence of the numerical method as reported in [9.

\subsection{Discussion}

In this section, the comparison between the experimental data and the numerical results is presented 


\begin{tabular}{lccccc}
\hline Parameter & & Units & $\begin{array}{c}\text { Granusil } \\
\# 8\end{array}$ & $\begin{array}{c}\text { Granusil } \\
\# 20 / 30\end{array}$ & $\begin{array}{c}2: 1 \text { mixture of Granusil } \\
\# 110 \text { and } \# 250\end{array}$ \\
\hline Porosity & $\phi$ & {$[-]$} & 0.4 & 0.32 & 0.35 \\
Intrinsic permeability & $K$ & {$\left[\mathrm{~m}^{2}\right]$} & $\begin{array}{c}1 \times 10^{-9} \\
2.30 \times 10^{-10}\end{array}$ & $6.36 \times 10^{-14}$ \\
Pore size distribution index & $\lambda$ & {$[-]$} & 4.275 & 7.33 & 5.35 \\
Entry pressure & $p_{d}$ & {$[\mathrm{~Pa}]$} & 600 & 1200 & 8027 \\
Residual liquid phase saturation & $S_{r, \ell}$ & {$[-]$} & 0.084 & 0.084 & 0.17 \\
Residual gas phase saturation & $S_{r, g}$ & {$[-]$} & 0 & 0 & 0 \\
\hline
\end{tabular}

TABLE 3. Material properties.

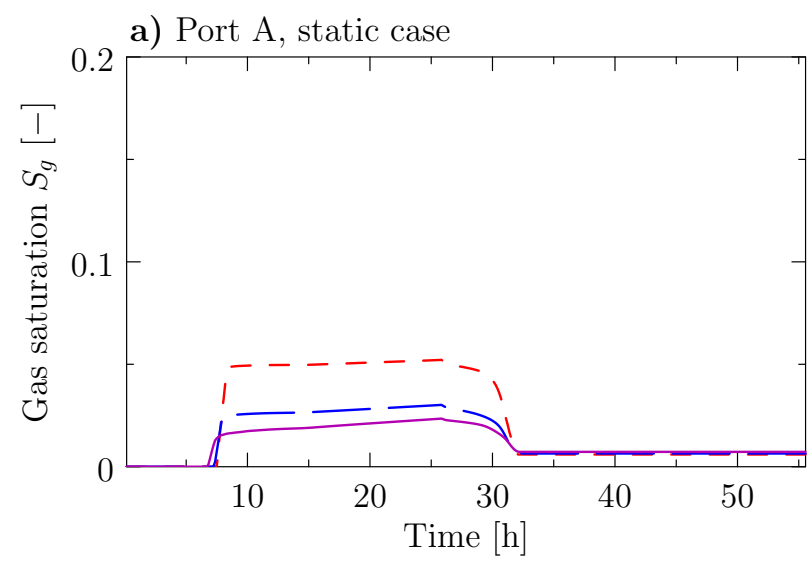

b) Port A, background flow

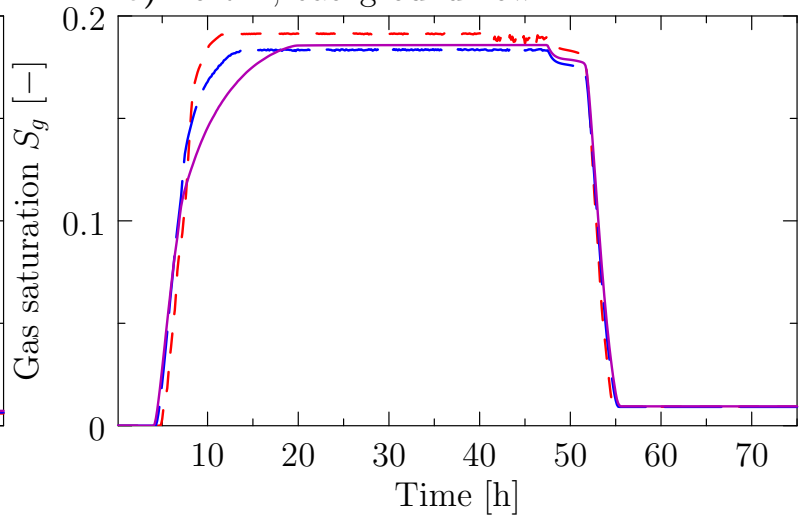

c) Port B, static case

d) Port B, background flow
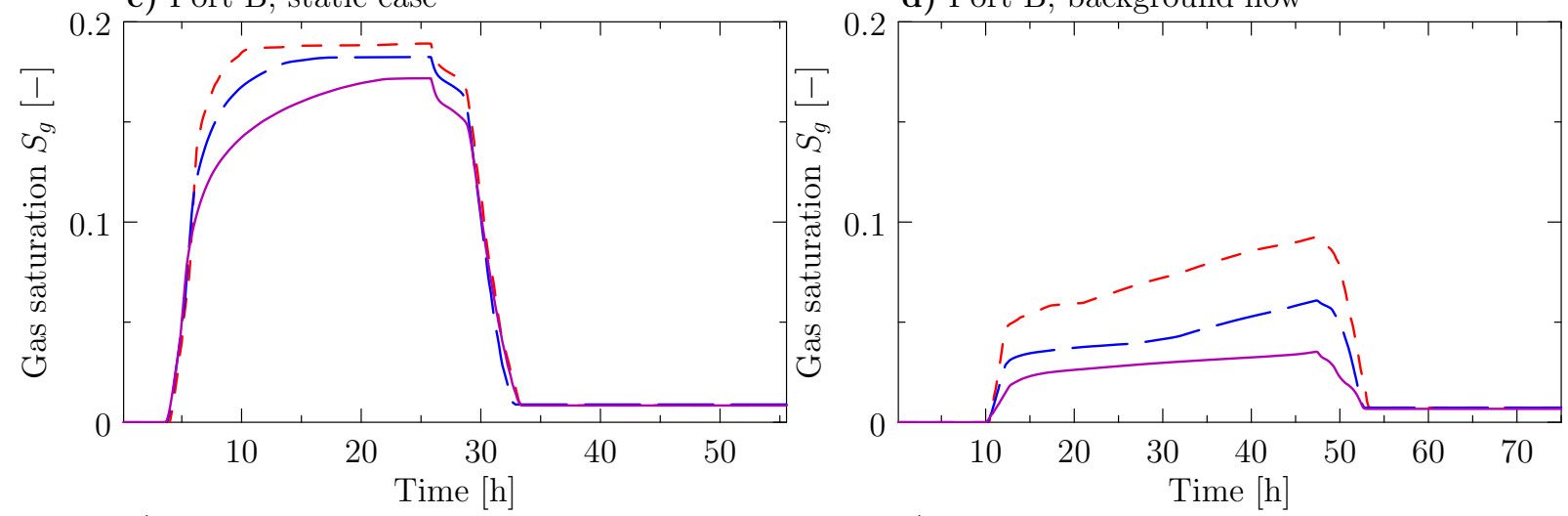

e) Port C, static case

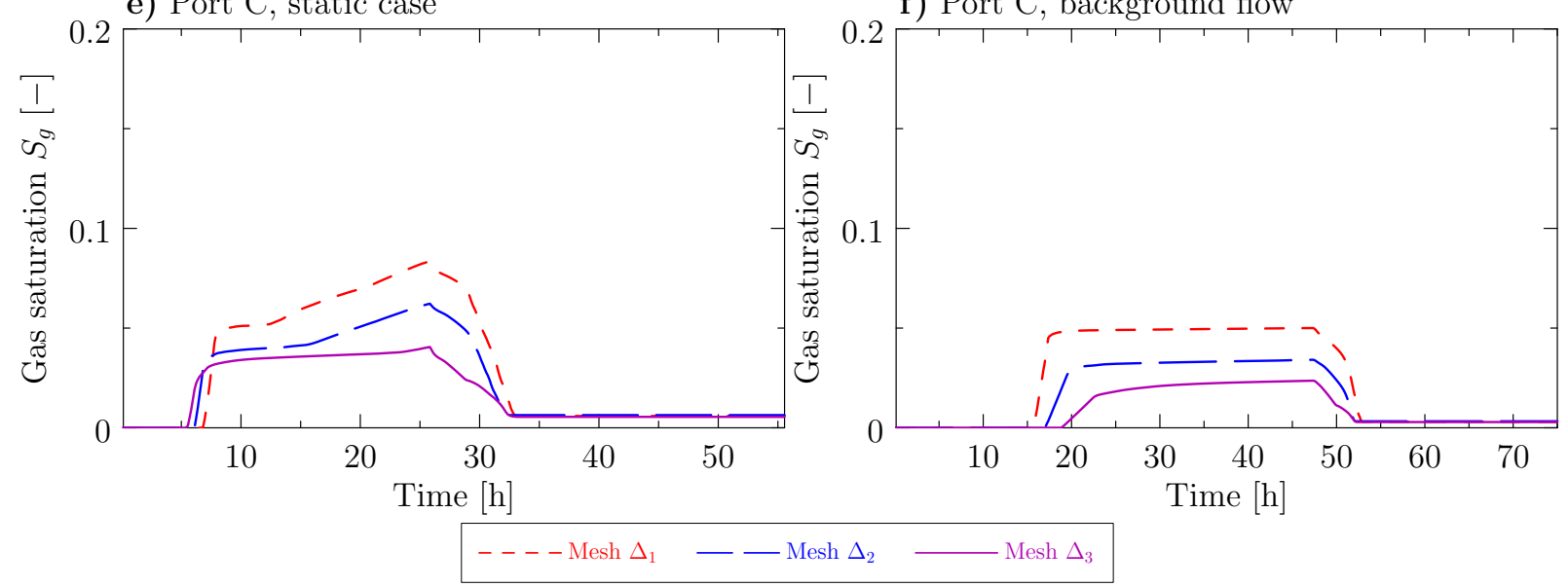

FIGURE 3. Illustration of mesh resolution effects on the numerical solution of the gas saturation evolution for $k=0.5 \mathrm{~s}^{-1}$ and $S_{c}=0.2$ at the sampling ports A (a b), B (c, d), and C (e, f) for both static (a, c, e) and background flow cases $(b, d, f)$. The mesh properties are given in Table 2 
a) Port A, static case

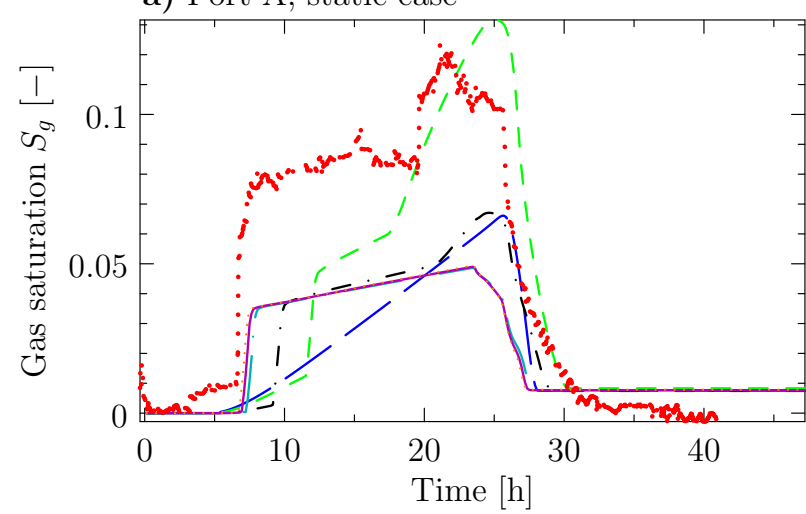

c) Port B, static case b) Port A, background flow

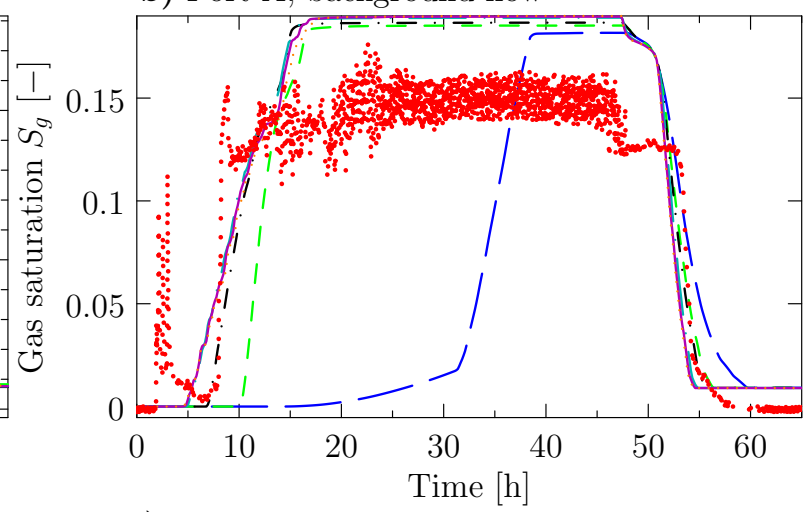

d) Port B, background flow
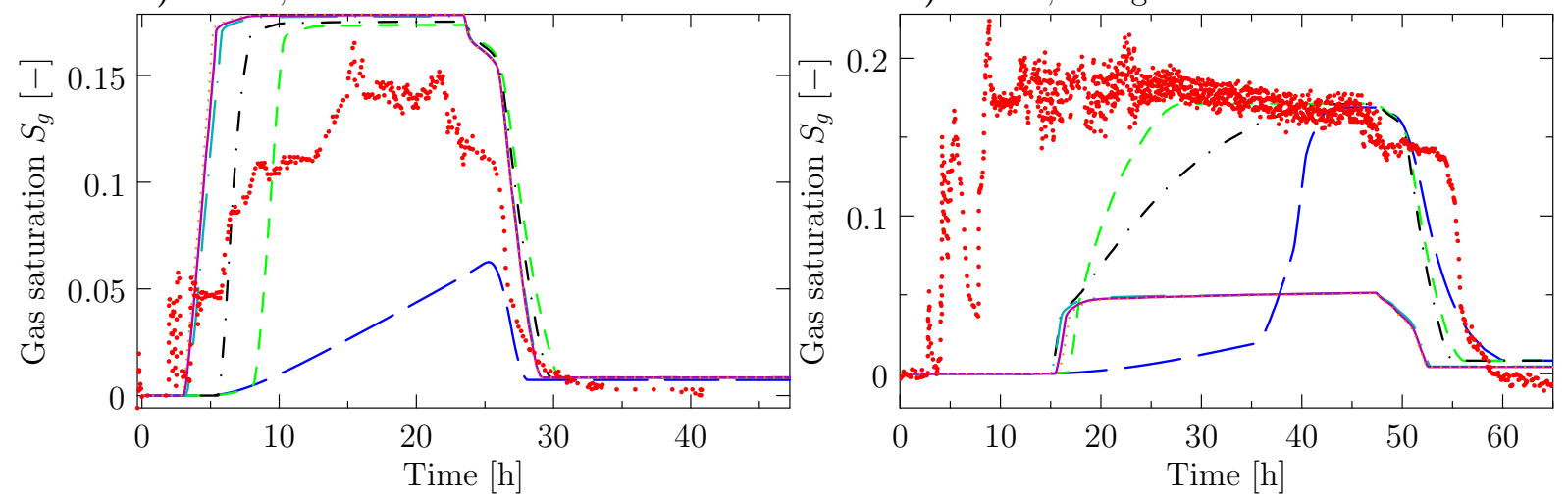

e) Port C, static case

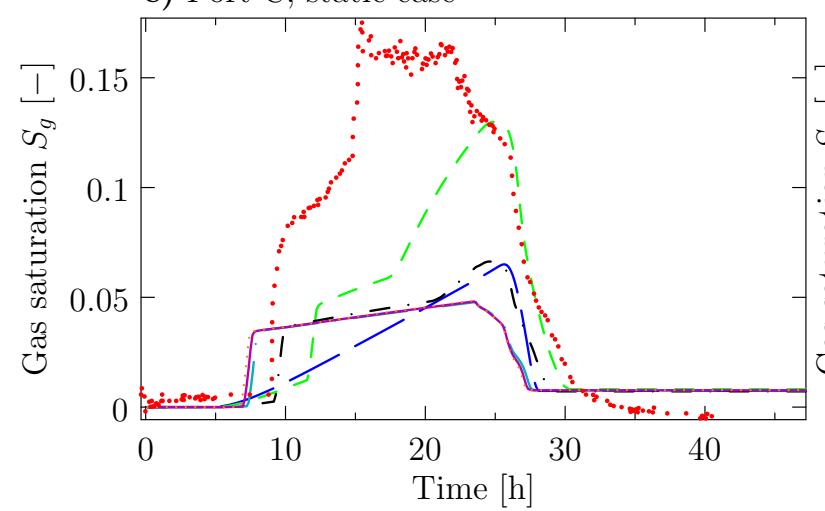

f) Port C, background flow

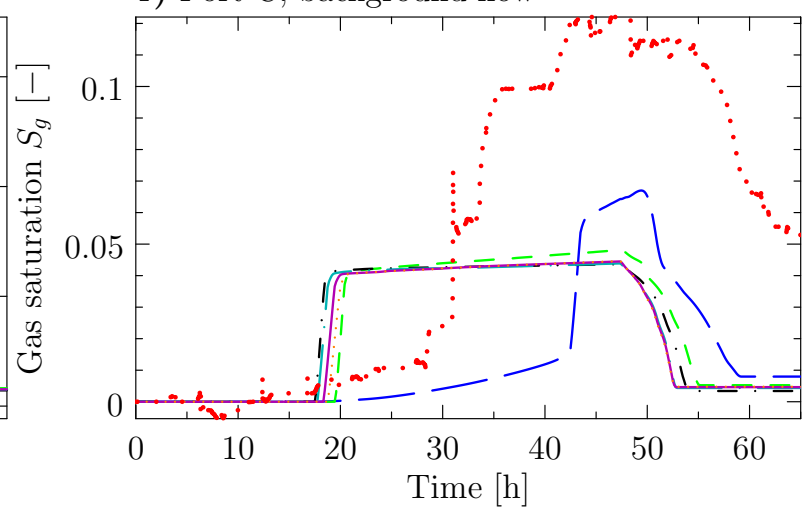

FiguRE 4. Results of the experiments and numerical simulations for (a) the static case and (b) the case with background flow. 


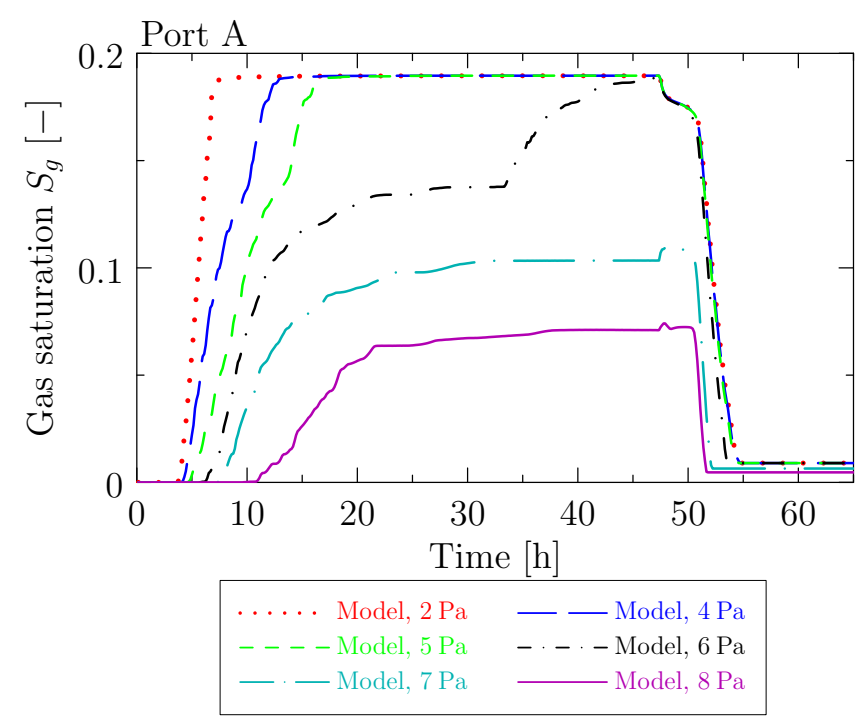

FiguRE 5. Results of the simulations performed with various pressure differences applied across the tank. The experiment with background flow corresponds to the simulation with a pressure difference of $5 \mathrm{~Pa}$.

and the mass transfer and accumulation processes are discussed for both static and background flow cases.

Figure 4 shows the results of gas saturation from the experiments and simulations for the static case and the case with background flow. The experimental data shown in (a) is from port B (directly above the injection port, as $\mathrm{CO}_{2}$-saturated water was injected into the left injection port in this case), while that in (b) is from port A (also above the injection port, as $\mathrm{CO}_{2}$-saturated water was injected into the right injection port in this case). Both experiments show the accumulation of gas phase $\mathrm{CO}_{2}$, then re-dissolution during the injection of clean water after the $\mathrm{CO}_{2}$ saturations had stabilized.

The simulation results for various choices of $k$ show that the best match with experimental data is achieved for rather large values of the mass transfer coefficient and that the simulated results are almost the same for those greater than a certain threshold value of 0.1 to $0.5 \mathrm{~s}^{-1}$. This indicates that the mass transfer processes are at near-equilibrium and that the background flow is not strong enough to substantially affect the $\mathrm{CO}_{2}$ exsolution and dissolution rates in these cases. On the other hand, the numerical results show that the presence of the background flow slows down the accumulation dynamics below the fine layer as indicated by different slopes of the saturation curves in Figure $4 c$ compared to Figure $4 \mathrm{~b}$. Note that because different injection ports were used in each experiment, the corresponding ports are shifted by one, i.e., results from port B for the static case are compared to the results from port $\mathrm{A}$ for the background flow case (see the gas saturation and $\mathrm{CO}_{2}$ mass fraction spatial evolution shown in Figures 6 and 7 ).

Further investigation of the background flow effect on the exsolution process can be done by analyzing the results of the simulations performed with different background flow rates, as shown in Figure 5 . The re- sults show a wide range of different gas accumulation behaviors, with higher flow rates leading to lower and slower exsolution due to different gas phase distribution around the injection ports. This indicates that the flow field significantly affects the multiphase $\mathrm{CO}_{2}$ evolution, however, the experimental data for these scenarios would be needed to quantify the impact.

While the analysis above indicated that nearequilibrium behavior occurred in the experiments, further experimental data are needed to determine generally in which cases the equilibrium mass transfer simplification is valid. Both experiments considered in this work are on a small scale and the measurement ports are close to the injection. Also, the difference between the static and background flow cases is not very substantial. Addressing these remaining knowledge gaps is one of the primary goals in [11, where non-equilibrium mass transfer behavior is observed in a large laboratory-scale system with background flow, and fundamentally different results are observed as compared with predominantly one-dimensional flows. This indicates that, for the decision between the kinetic and equilibrium models, flow field and scale both need to be taken into account. Additionally, the flow field and scale are not the only factors controlling the multiphase evolution, as also indicated in [11, which showed that temperature fluctuations can also significantly affect mass transfer processes.

\section{Conclusions}

To assess the potential risks of groundwater contamination via leakage of stored $\mathrm{CO}_{2}$ from deep geologic carbon sequestration sites, we must be able to predict multiphase $\mathrm{CO}_{2}$ transport through shallow aquifers. To aid in this effort, an innovative numerical model was applied and tested against data from well-controlled, small-scale laboratory experiments. Beyond conventional continuum-based two-phase flow 
Gas saturation $S_{q}$, static case

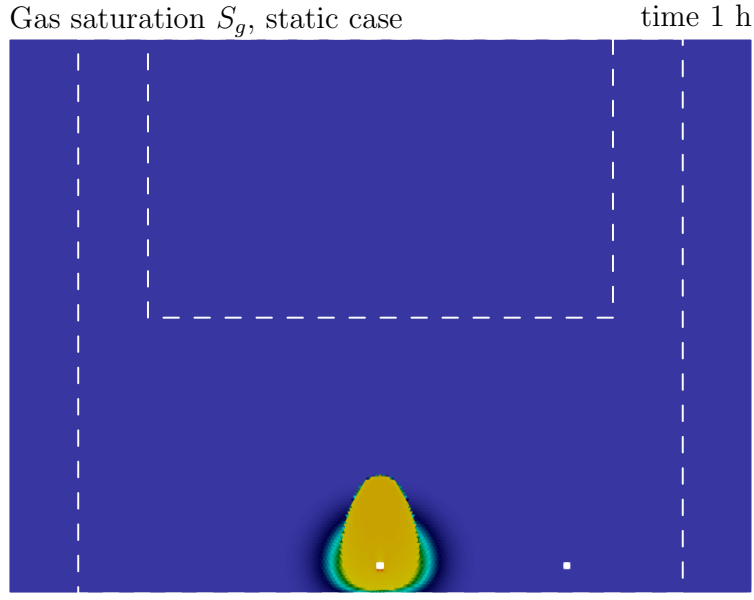

Gas saturation $S_{g}$, static case

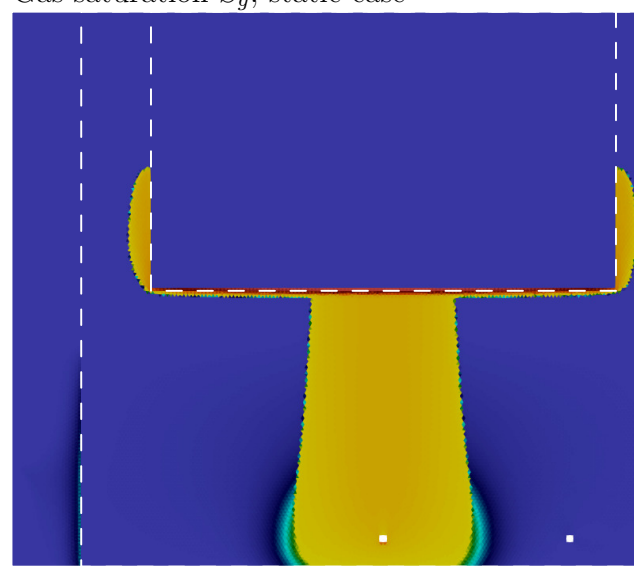

Gas saturation $S_{g}$, static case

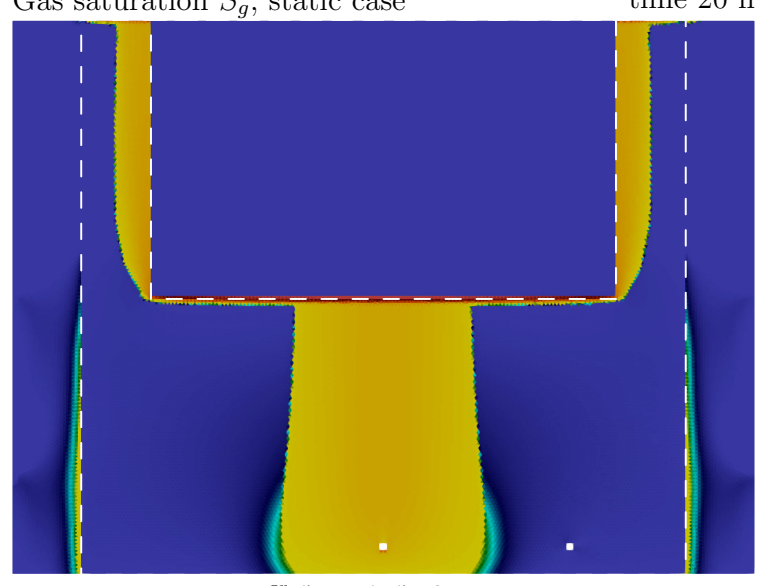

Effective gas saturation $s^{5}$

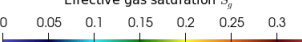

time $10 \mathrm{~h}$ Mass fraction $X$, static case
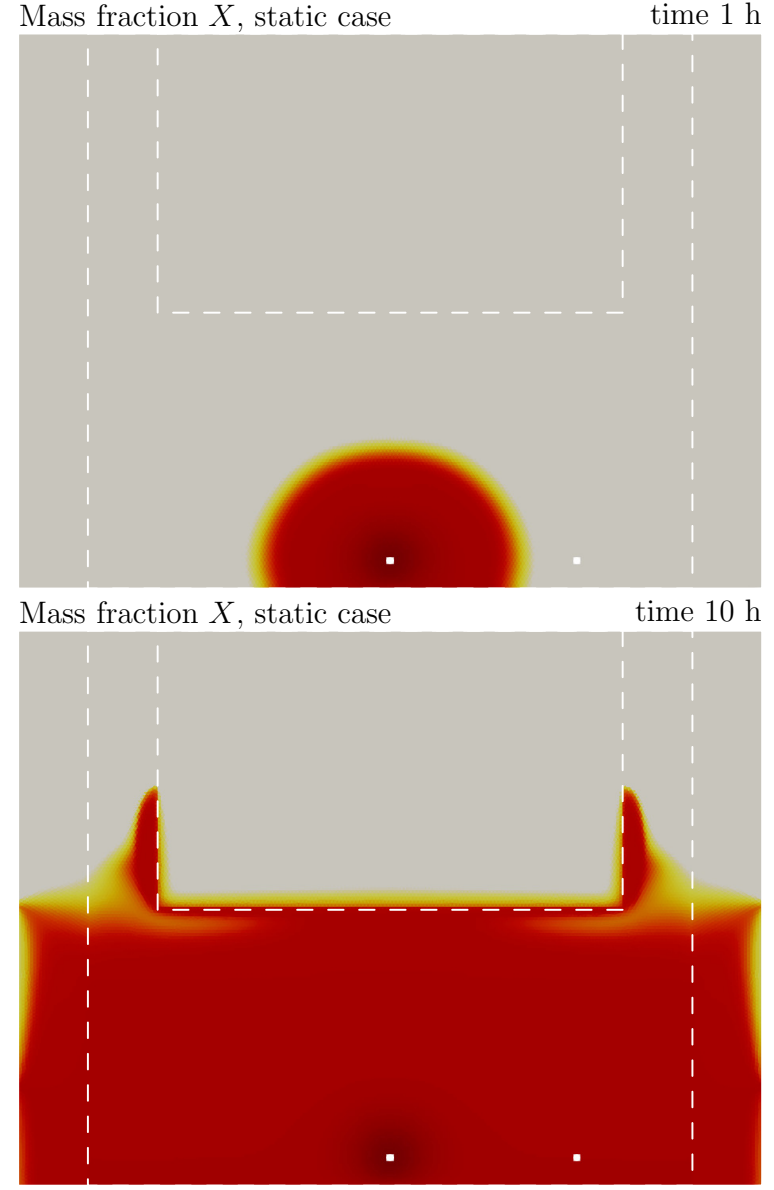

Mass fraction $X$, static case

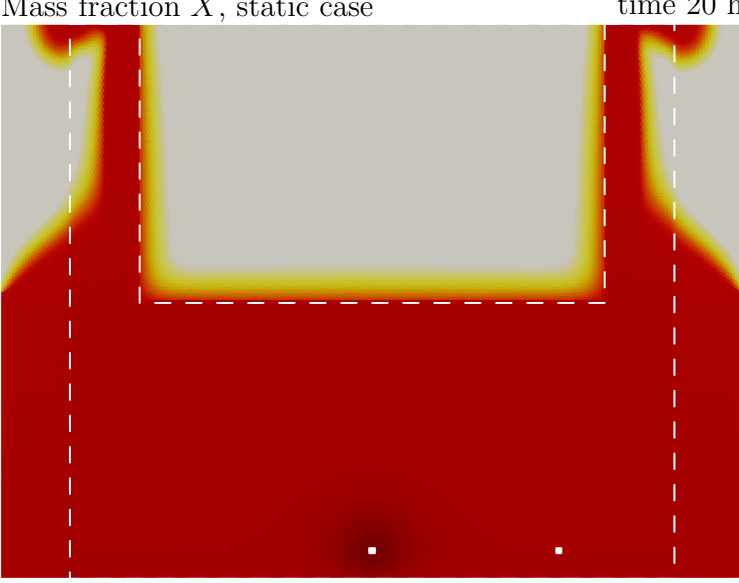

$\mathrm{CO}_{2}$ mass fraction $X$

$\begin{array}{llllllll}0 & 0.0002 & 0.0004 & 0.0006 & 0.0008 & 0.001 & 0.0012\end{array}$

Figure 6. Spatial distribution of $S_{g}$ (left) and $X$ (right) for the static case at $1 \mathrm{~h}, 10 \mathrm{~h}$, and $20 \mathrm{~h}$ (from top to bottom), computed using $k=0.5 \mathrm{~s}^{-1}$ and $S_{c}=0.2$. 


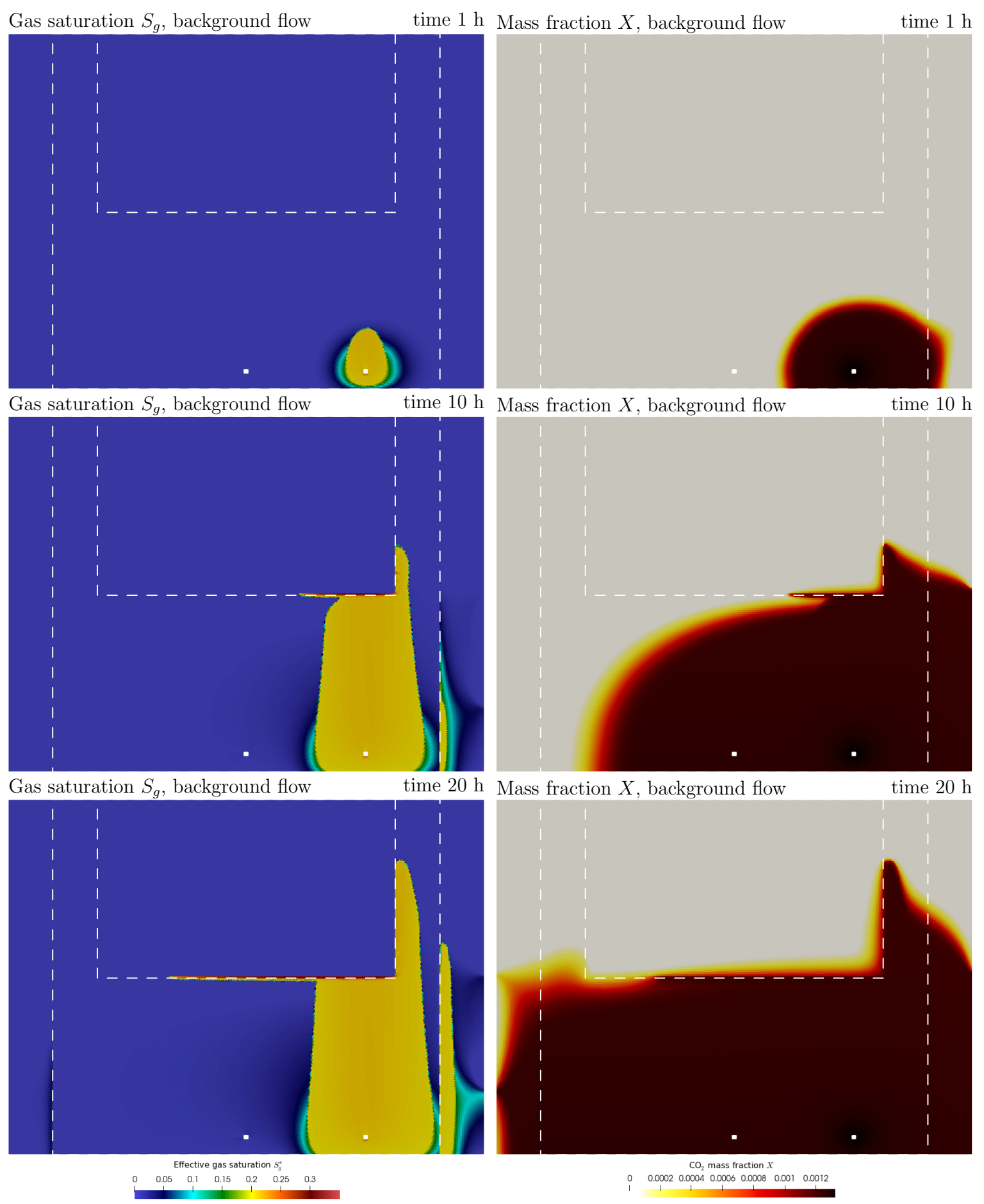

Figure 7. Spatial distribution of $S_{g}$ (left) and $X$ (right) for the background flow case at $1 \mathrm{~h}, 10 \mathrm{~h}$, and $20 \mathrm{~h}$ (from top to bottom), computed using $k=0.5 \mathrm{~s}^{-1}$ and $S_{c}=0.2$. 
in porous media, the model incorporated the ability to simulate non-equilibrium $\mathrm{CO}_{2}$ mass transfer between the aqueous and gaseous phases during exsolution and dissolution processes.

The experimental data used in this work clearly showed equilibrium or near-equilibrium behavior for both steady and background flow cases. These findings are in contrast with the non-equilibrium behavior observed on a larger scale for the scenarios with background flow. This indicates that the mass transfer for the similar scenarios is not controlled by the flow field only. Further investigation of the mass transfer is needed to understand for which scenarios the kinetic model is needed and when the equilibrium assumption is sufficient. The computational methodology developed here could be used to assess both equilibrium and non-equilibrium processes in these future scenarios.

\section{ACKnOWLEDGMents}

The work reported in this paper was supported by the Ministry of Education, Youth and Sports of the Czech Republic under the OP RDE grant number CZ.02.1.01/0.0/0.0/16_019/0000778 Centre for Advanced Applied Sciences and Inter-Excellence grant no. LTAUSA19021. Any use of trade, firm, or product names in this publication is for descriptive purposes only and does not imply endorsement by the U.S. Government.

\section{REFERENCES}

[1] S. Pacala, R. Socolow. Stabilization wedges: solving the climate problem for the next 50 years with current technologies. Science 305(5686):968-972, 2004. DOI:10.1126/science. 1100103

[2] J. A. Apps, L. Zheng, Y. Zhang, et al. Evaluation of potential changes in groundwater quality in response to CO2 leakage from deep geologic storage. Transport in Porous Media 82(1):215-246, 2010. DOI:10.1007/s11242-009-9509-8

[3] M. R. Plampin, T. H. Illangasekare, T. Sakaki, R. J. Pawar. Experimental study of gas evolution in heterogeneous shallow subsurface formations during leakage of stored CO2. International Journal of Greenhouse Gas Control 22:47-62, 2014. DOI:10.1016/j.ijggc.2013.12.020

[4] M. R. Plampin, R. N. Lassen, T. Sakaki, et al. Heterogeneity-enhanced gas phase formation in shallow aquifers during leakage of co2-saturated water from geologic sequestration sites. Water Resources Research 50(12):9251-9266, 2014. DOI:10.1002/2014WR015715

[5] T. Sakaki, M. R. Plampin, R. Pawar, et al. What controls carbon dioxide gas phase evolution in the subsurface? experimental observations in a $4.5 \mathrm{~m}$-long column under different heterogeneity conditions. International Journal of Greenhouse Gas Control 17:66-77, 2013. DOI:10.1016/j.ijggc.2013.03.025.

[6] M. R. Plampin, M. L. Porter, R. J. Pawar, T. H. Illangasekare. Intermediate-scale experimental study to improve fundamental understanding of attenuation capacity for leaking $\mathrm{CO} 2$ in heterogeneous shallow aquifers. Water Resources Research 53(12):10121-10138, 2017. DOI:10.1002/2016WR020142

[7] M. L. Porter, M. Plampin, R. Pawar, T. Illangasekare. $\mathrm{CO} 2$ leakage in shallow aquifers: A benchmark modeling study of $\mathrm{CO} 2$ gas evolution in heterogeneous porous media. International Journal of Greenhouse Gas Control 39:51-61, 2015. DOI:10.1016/j.ijggc.2015.04.017

[8] R. N. Lassen, M. R. Plampin, T. Sakaki, et al. Effects of geologic heterogeneity on migration of gaseous CO2 using laboratory and modeling investigations. International Journal of Greenhouse Gas Control 43:213-224, 2015. DOI:10.1016/j.ijggc.2015.10.015

[9] R. Fučík, J. Klinkovský, J. Solovský, et al. Multidimensional mixed-hybrid finite element method for compositional two-phase flow in heterogeneous porous media and its parallel implementation on gpu. Computer Physics Communications 238:165-180, 2019. DOI:10.1016/j.cpc.2018.12.004.

[10] M. R. Plampin, M. Porter, R. Pawar, T. H. Illangasekare. Multi-scale experimentation and numerical modeling for process understanding of co2 attenuation in the shallow subsurface. Energy Procedia 63:4824-4833, 2014. DOI:10.1016/j.egypro.2014.11.513.

[11] J. Solovský, R. Fučík, M. R. Plampin, et al. Dimensional effects of inter-phase mass transfer on attenuation of structurally trapped gaseous carbon dioxide in shallow aquifers. Journal of Computational Physics 40, 2020. DOI:10.1016/j.jcp.2019.109178.

[12] P. Bastian. Numerical Computation of Multiphase Flows in Porous Media. Habilitation thesis, Kiel University, 2000.

[13] R. Helmig. Multiphase Flow and Transport Processes in the Subsurface, A contribution to the Modelling of Hydrosystems. Springer, 1997.

[14] K. Mosthaf, K. Baber, B. Flemisch, et al. A coupling concept for two-phase compositional porous-medium and single-phase compositional free flow. Water Resources Research 47(10), 2011. DOI:10.1029/2011WR010685

[15] R. Brooks, A. Corey. Hydraulic Properties of Porous Media. Colorado State University, Hydrology Paper 3:27, 1964.

[16] N. Burdine. Relative permeability calculations from pore size distribution data. Journal of Petroleum Technology 5:71-78, 1953. DOI:10.2118/225-G

[17] I. Tsimpanogiannis, Y. C. Yortsos. The critical gas saturation in a porous medium in the presence of gravity. J Colloid Interface Sci 270:388-395, 2014. DOI:10.1016/j.jcis.2003.09.036

[18] R. J. Millington, J. P. Quirk. Permeability of porous solids. Transactions of the Faraday Society 57:1200-1207, 1961.

[19] J. Niessner, S. M. Hassanizadeh. Modeling kinetic interphase mass transfer for two-phase flow in porous media including fluid-fluid interfacial area. Transport in Porous Media 80:329-344, 2009.

DOI:10.1007/s11242-009-9358-5 
[20] R. Sander. Compilation of Henry's Law Constants for Inorganic and Organic Species of Potential Importance in Environmental Chemistry. Max-Planck Inst of Chem, Mainz, Germany 1999.

[21] C. Geuzaine, J.-F. Remacle. Gmsh: A 3-D finite element mesh generator with built-in pre-and post-processing facilities. International Journal for Numerical Methods in Engineering 79(11):1309-1331, 2009. DOI:10.1002/nme.2579
[22] J. Solovský, R. Fučík. Mass Lumping for MHFEM in Two Phase Flow Problems in Porous Media. In F. A. Radu, K. Kumar, I. Berre, et al. (eds.), Numerical Mathematics and Advanced Applications ENUMATH 2017, pp. 635-643. Springer International Publishing, 2019. DOI:10.1007/978-3-319-96415-7_58

[23] J. Solovský, R. Fučík. A Parallel mixed-hybrid finite element method for two phase flow problems in porous media using MPI. Computer Methods in Materials Science 17:84-93, 2017. 